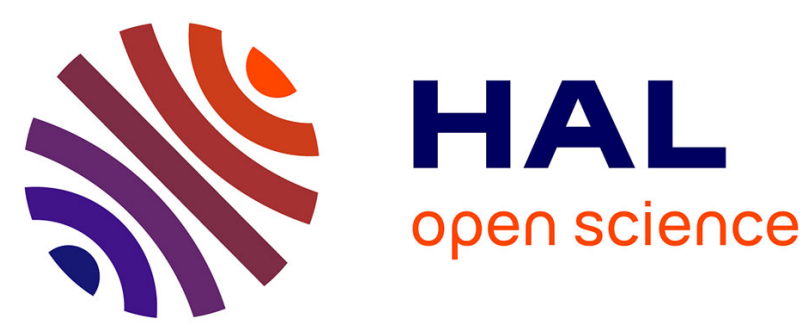

\title{
A standardized Method to Automatically Segment Amyloid Plaques in Congo Red Stained Sections from Alzheimer Transgenic Mice.
}

Olivier Teboul, Abdelmonem Feki, Albertine Dubois, Bruno Bozon, Alexis Faure, Philippe Hantraye, Marc Dhenain, Benoit Delatour, Thierry Delzescaux

\section{To cite this version:}

Olivier Teboul, Abdelmonem Feki, Albertine Dubois, Bruno Bozon, Alexis Faure, et al.. A standardized Method to Automatically Segment Amyloid Plaques in Congo Red Stained Sections from Alzheimer Transgenic Mice.. Conf Proc IEEE Eng Med Biol Soc, 2007, 1, pp.5593-5596. 10.1109/IEMBS.2007.4353614 . hal-00320530

\section{HAL Id: hal-00320530 \\ https://hal.science/hal-00320530}

Submitted on 25 May 2021

HAL is a multi-disciplinary open access archive for the deposit and dissemination of scientific research documents, whether they are published or not. The documents may come from teaching and research institutions in France or abroad, or from public or private research centers.
L'archive ouverte pluridisciplinaire HAL, est destinée au dépôt et à la diffusion de documents scientifiques de niveau recherche, publiés ou non, émanant des établissements d'enseignement et de recherche français ou étrangers, des laboratoires publics ou privés. 


\title{
A standardized Method to Automatically Segment Amyloid Plaques in Congo Red Stained Sections from Alzheimer Transgenic Mice
}

\author{
Olivier Teboul and Abdelmonem Feki and Albertine Dubois \\ and Bruno Bozon and Alexis Faure and Philippe Hantraye and \\ Marc Dhenain and Benoit Delatour and Thierry Delzescaux
}

\begin{abstract}
Automated detection of amyloid plaques (AP) in post mortem brain sections of patients with Alzheimer disease (AD) or in mouse models of the disease is a major issue to improve quantitative, standardized and accurate assessment of neuropathological lesions as well as of their modulation by treatment. We propose a new segmentation method to automatically detect amyloid plaques in Congo Red stained sections based on adaptive thresholds and a dedicated amyloid plaque/tissue modelling. A set of histological sections focusing on anatomical structures was used to validate the method in comparison to expert segmentation.
\end{abstract}

\section{INTRODUCTION}

Alzheimer disease $(\mathrm{AD})$ is a progressive neurodegenerative disorder that affects a large proportion of the elderly population [1]. One of the characteristic of histological sections from the brain of patients who had the disease is the presence of many amyloid plaques. These lesions are extracellular deposits mainly made of amyloid protein. They can be detected after histological processing such as tissue staining by the Congo Red method. Each plaque measures approximately from 20 to $200 \mu \mathrm{m}$. Transgenic mice presenting amyloid plaques (AP) are widely studied to improve our understanding of the pathophysiology of AD but also to investigate new therapeutics. The automated detection of amyloid plaques on large dataset of histological sections remains mainly manual or semiautomatic and based on basic image processing methods using histogram or color image analysis [2]. More recently, an approach using statistical model based on prior operator expertise has been proposed [3]. Nevertheless, most of these approaches remain tedious and time consuming.

In this paper, we propose a fully automated method to detect amyloid plaques in Congo Red stained sections of transgenic mouse brains. The first step consisted in the separation of the tissues from the background based on an expectation maximization (EM) algorithm followed by the extraction of the seeds corresponding to the plaques using global/local adaptive thresholds. The second step exploited

O. Teboul, A. Feki, A. Dubois, M. Dhenain, P. Hantraye and T. Delzescaux are with the Medical Image Research Center (MIRCen), URA CEA-CNRS 2210, 4 place du Général Leclerc, 914006 Orsay Cedex, France thierry.delzescaux@cea.fr

B. Bozon, A. Faure and B. Delatour are with the NAMC, CNRS UMR 8620, Bt 446 Universit Paris-Sud, 1405 Orsay Cedex, France benoit.delatour@u-psud.fr the seed and the corresponding region previously extracted to fit a mathematical model estimating iteratively a plane with uniform variance for the tissues and a gaussian surface for the amyloid plaques. The results were then filtered using an a priori area and shape constraints. The processes were implemented in 2-D and could be extented to series of stacked sections (3-D volume). Several informative parameters such as amyloid load, plaque number, area, distribution and $\mathrm{R}$, G, B intensities could be robustly and reproducibly assessed from the samples with the proposed method.

\section{MATERIALS AND METHODS}

\section{A. Biological materials}

During our studies, image analysis were performed on histological sections from hemibrains of APP/PS1 and APP/PS1KI mouse models of AD. The brains were cut into $40 \mu \mathrm{m}$-thick coronal (anisotropic uniform random) sections on a freezing microtome. Brain sections were stained by the Congo Red method. All sections were then digitized using a Nikon Coolscan 4000 ED scanner as RGB images with a 4000 dpi in-plane digitization resolution (pixel size 6.35e$\left.3 \times 6.35 \mathrm{e}-3 \mathrm{~mm}^{2}\right)$ generating large images $(1300 * 1300$ pixels)(Fig.1a). Image analysis was conducted during two successive experiments. First, the method was tested in selected brain regions (hippocampus (HP) and frontal (FR) part of the brain manually delineated) from 5 APP/PS1 and 5 APP/PS1KI mice. The APP/PS1 mice displayed large amyloid plaques (Fig.1b, 1c) while plaques were smaller in APP/PS1KI animals (Fig.1d, 1e). This experiment was conducted to evaluate the robustness of automatic detection under a variety of histological contexts. In a second analysis, plaque detection was evaluated from histological sections that covered the whole brain of a single APP/PS1 animal. This allowed to extend the study to different brain regions. For this experiment, one histological brain section out of twelve was extracted so as to finally keep 13 sections with an associated inter-slices thickness of $0.48 \mathrm{~mm}$.

\section{B. Overall protocol}

From RGB images, G color component was extracted and corresponding gray scales were inverted. This channel is predominantly used in histological image processing due to the lower level of noise and its high natural contrast [4]. A binary mask of the brain section was computed with 
EM procedure [5] which estimated a threshold value under hypothesis that intensities of the background and the tissue can be assimilated to gaussian distributions.

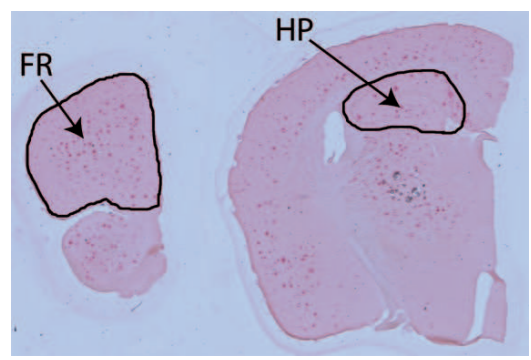

a)

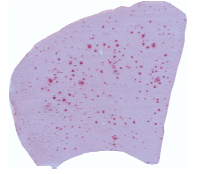

b)

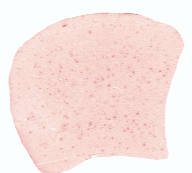

d)

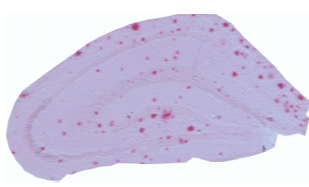

c)

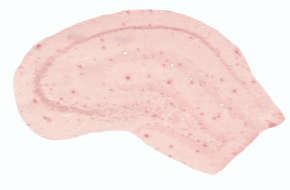

e)
Fig. 1. a) Coronal sections stained using Congo Red corresponding to frontal (FR) and hippocampal (HP) locations. Frontal and hippocampic regions extracted respectively from APP-PS1 (resp. b) et c) ) and APPPS1-KI (resp. d) and e) ).

The definition of seeds corresponding roughly to amyloid plaques (AP) was performed in a 2-steps scheme. To discriminate plaques from tissue, we assumed that tissue was the majority class in the section. For a region of interest defined (mask and corresponding image), mean $m$ and standard deviation $\sigma$ values were calculated and pixels presenting a value over $T=m+\sigma$ were automatically outlined and labelled as seeds. To select the optimal contrast between plaques and tissue stainings, this approach was applied twice: 1) on the global masked section to generate a rough estimation of the seeds which was used to create a generalized Voronoi's partition of the image domain [6], [7] and 2) on each Voronoi's partition previously obtained to refine both seeds estimation and consecutively second Voronoi's partitions. Based on these results, we have proposed a mathematical modelling able to segment AP.

\section{Mathematical model}

The divide-and-conquer strategy that has been chosen lead to an easier segmentation problem: separating a plaque $(\mathcal{P})$ from the tissue $(\mathcal{T})$. As a consequence, we introduced a model for each class (tissue, AP). The classification algorithm worked in two steps: estimate the parameters of the models and classify the pixels until convergence.

1) Plaque Model: We were looking for an amyloid plaque, which is actually a load of amyloids. As the Congo
Red stains these proteins, the intensity of Congo Red is proportional to the density of amyloid. Thus, we considered the intensity of Congo Red in a pixel as a mesure of the amyloid load in this pixel's area. Moreover, we supposed that in a plaque, the proteins were distributed according to a spatial gaussian law around the center of the plaque:

$$
p(X \mid \mathcal{P})=\frac{1}{\alpha} \exp \left(-\frac{1}{2}\left(X-\mu_{p}\right)^{T} \Sigma_{p}^{-1}\left(X-\mu_{p}\right)\right)
$$

where $\alpha=2 \pi \operatorname{det}\left(\Sigma_{p}\right)^{1 / 2}$ and $X=(x, y)^{T}$

This meant that the location $X$ of a amyloid protein was a random variable which probability was $p(X)$. Besides, we assume that the observed intensity $I$ in that location $X$ matched with $I$ observations of that random variable.

However, we did not observe only amyloid protein stains with Congo Red but also the brain tissues which had to be taken into account.

2) Tissue Model: We assumed that the brain intensity followed a gaussian law:

$$
p(I \mid \mathcal{T})=\frac{1}{\sqrt{2 \pi} \sigma_{b}} \exp \left(-\frac{\left(I-\mu_{b}\right)^{2}}{2 \sigma_{b}^{2}}\right)
$$

This meant that the probability to observe an intensity $I$ in the tissue was independant of the location, and was equal to $p(I)$. The main difficulty of this formulation was that the observations didn't belong to the same spaces: in the plaque model, the observations were the locations of proteins, and in the second one, the observations were the pixels intensities. As a consequence, we used an heuristic to classify the pixels between the two classes, based upon the posterior probabilities of the pixels to belong to these two classes. The algorithm worked in two steps: estimate and classify.

3) Parameters estimation: The estimation of the parameters was based upon the maximum likelihood of the two classes.

$$
\begin{array}{r}
\mu_{b}=\frac{\sum_{n}\left(1-\tau_{n}\right) I_{n}}{\sum_{n}\left(1-\tau_{n}\right)} \\
\sigma_{b}^{2}=\frac{\sum_{n}\left(1-\tau_{n}\right)\left(I_{n}-\mu_{b}\right)^{2}}{\sum_{n}\left(1-\tau_{n}\right)} \\
\mu_{p}=\frac{\sum_{n} \tau_{n} I_{n} X_{n}}{\sum_{n} I_{n} \tau_{n}} \\
\Sigma_{p}=\frac{\sum_{n} \tau_{n} I_{n}\left(X_{n}-\mu_{p}\right)\left(X_{n}-\mu_{p}\right)^{T}}{\sum_{n} I_{n} \tau_{n}}
\end{array}
$$

where $\tau=1$ if the pixel was in the plaque and $\tau=0$ otherwise.

4) Classification: In order to classify a pixel, we computed its posterior probability (score $S$ ) in the two classes using $(1,2)$ and the prior probability of each class. We labelized the pixel by comparing the two scores. For instance, if a pixel had an intensity close to the tissue intensity, the score for the tissue class was high (same intensity) whereas the score for the plaque class was low (because it was far from the plaque center).

$$
\begin{aligned}
S_{p}\left(v_{i}\right) & =\pi p\left(X_{i} \mid \mathcal{P}\right) \\
S_{t}\left(v_{i}\right) & =(1-\pi) p\left(I_{i} \mid \mathcal{T}\right)
\end{aligned}
$$


where $S_{p}\left(v_{i}\right)$ and $S_{t}\left(v_{i}\right)$ were the scores of the voxel $v_{i}$ with respect to both classes.

\section{Data analysis}

The amyloid load which was the ratio between the quantity of amyloid plaques and the tissue was the main information to be calculated to characterize AD-related pathology. Due to the processing on 2-D data, we assumed that the ratio of the surfaces was proportional to the ratio of the volume enabling us to assess amyloid load for both individual sections (validation part) and series of sections (whole brain) according to the principle of Delesse validated in stereology. In addition, shape, position features and intensity of each individual plaque were also computed.

In a first analysis, the proposed methodology was applied to a subset of 20 images focusing on two anatomical structures manually delineated by an expert: the hippocampus (HP) $(n=10)$ and the frontal (FR) region $(n=10)$ including both APP/PS1 (large plaques) and APP/PS1KI mice (small plaques). The computation of the amyloid load of reference for each region was performed by a biologist expert using Visilog 6 software (Nosis, Les Ulis, France) and a dedicated image processing protocol (R, G, B color component adjustment, global automated threshold based on entropy criterion and morphometric filtering according to Feret's diameter). A correlation analysis was performed to test the linear relationship between the measurements of the two methods. The second part of the work consisted in analysing a mouse brain (representing 13 sections) in order to test a whole section analysis and assess information in 3-D. In the end, visual inspection of the results based on a volume rendering of the plaques in 3-D was performed.

\section{RESULTS}

Figure 2 describes the sequential results obtained at different step of the AP segmentation in hippocampus of an APP-PS1 mouse (Fig.2a). The automated extraction of the hippocampus was performed on the inverted green color component (Fig.2b).

The definition of subregions including a single seed was performed in a 2-steps process: the first one provided a rough estimation of the seeds which generated several large Voronoi's partitions coded with a Rainbow colormap (Fig.2c) while the second step produced an oversegmentation leading to small and numerous Voronoi's partitions (Fig.2d). This information was then treated with the mathematical model proposed in order to filter real AP from artefacts (border, dust, vessels, stria) and to assess quantitative parameters (location, area, intensity). We proposed a pseudo 3D representation of the detected AP as spheres (presenting a size and a color texture relative to their mean radius) which were superimposed on the mask of the hippocampus (Fig.2e). Masked plaques were also superimposed to the original image to visually appreciate the reliability of the method (Fig.2f).

In order to validate it, this methodology was applied to two selected brain regions (HP and FR) from mice with

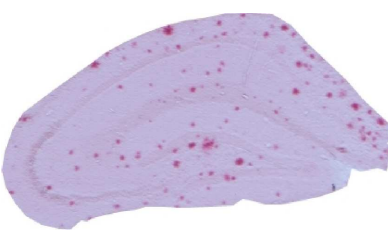

a)

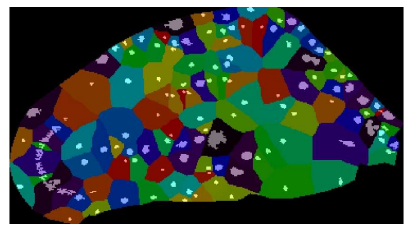

c)

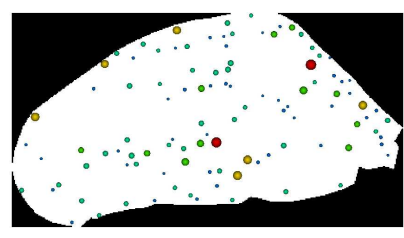

e)

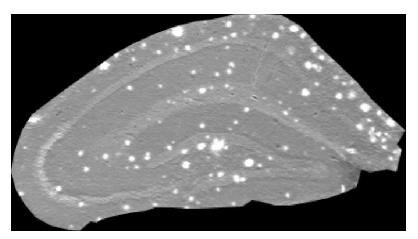

b)

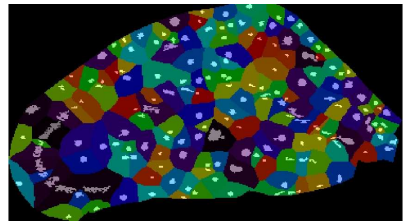

d)

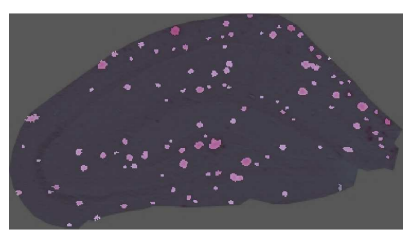

f)
Fig. 2. a) HP region stained using Congo Red in APP-PS1 mouse, b) inverted and masked green color component, c) fi rst and d) second seeds estimation and Voronoi computation. e) Final AP extracted with the automated method and f) image fusion of the original image with the detected AP (bright spots).

TABLE I

RESULTS OF AMYLOID LOAD COMPUTED BY THE EXPERT AND THE AUTOMATED METHODS ON HP AND FR REGIONS IN APP-PS1 AND APP-PS1-KI MICE.

\begin{tabular}{|c|c|c|c|c|c|c|}
\hline Mice (m) & Region/Method & $\mathrm{m} 1$ & $\mathrm{~m} 2$ & $\mathrm{~m} 3$ & $\mathrm{~m} 4$ & $\mathrm{~m} 5$ \\
\hline \multirow{4}{*}{ APP-PS1 } & FR (expert) & 3.8 & 4.1 & 5.0 & 4.1 & 4.0 \\
\cline { 2 - 7 } & FR (automated) & 5.6 & 4.5 & 5.4 & 4.6 & 5.3 \\
\cline { 2 - 7 } & HP (expert) & 3.6 & 3.5 & 4.8 & 4.3 & 4.4 \\
\cline { 2 - 7 } & HP (automated) & 4.4 & 4.3 & 5.1 & 5.0 & 5.0 \\
\hline APP-PS1-KI & FR (expert) & 2.2 & 1.6 & 1.2 & 1.2 & 1.4 \\
\cline { 2 - 7 } & FR (automated) & 2.7 & 2.1 & 1.9 & 2.1 & 2.0 \\
\cline { 2 - 7 } & HP (expert) & 1.6 & 0.9 & 1.3 & 0.8 & 1.4 \\
\cline { 2 - 7 } & HP (automated) & 2.9 & 1.8 & 2.6 & 2.1 & 2.7 \\
\hline
\end{tabular}

large and small amyloid plaques ( $\mathrm{n}=5$ and 5, respectively). Amyloid load values were estimated in the targeted anatomical regions (Table 1). Expert and automated methods had a highly and significant correlation: $R=0.96$, $p<0.0001$ (Fig.3).

A 3-D surface rendering view representing AP as spheres for the sections of a single brain is presented in figure 4 to highlight the ability of the method to deal with 3$\mathrm{D}$ data (stacked sections). To facilitate the vizualization, inter-section space was artificially increased to $2 \mathrm{~mm}$. It is interesting to notice that AP were mainly located in the cortical areas but also in internal structures like thalamus and hippocampus (section 10, Fig.4). 
